\title{
Current Status of Endoscopic Ultrasound Techniques for Pancreatic Neoplasms
}

\author{
Yousuke Nakai, Naminatsu Takahara, Suguru Mizuno, Hirofumi Kogure and Kazuhiko Koike \\ Department of Gastroenterology, Graduate School of Medicine, The University of Tokyo, Tokyo, Japan
}

Endoscopic ultrasound (EUS) now plays an important role in the management of pancreatic neoplasms. There are various types of pancreatic neoplasms, from benign to malignant lesions, and the role of EUS ranges from the imaging diagnosis to treatment. EUS is useful for the detection, characterization, and tissue acquisition of pancreatic lesions. Recent advancement of contrast-enhanced harmonic EUS and elastography enables better characterization of pancreatic lesions. In addition to these enhanced EUS imaging techniques, EUS-guided tissue acquisition is now the standard procedure to establish the pathological diagnosis of pancreatic neoplasms. While these diagnostic roles of EUS have been established, EUS-guided interventions such as ablation and drainage are also increasingly utilized in the management of pancreatic neoplasms. However, most of these EUS-guided interventions are not yet standardized in terms of techniques and devices and thus need further investigations. Clin Endosc 2019;52:527-532

Key Words: Endosonography; Fine needle aspiration; Pancreatic neoplasms

\section{INTRODUCTION}

Pancreatic cancer is now the fourth leading cause of cancer mortality, and its prognosis is still poor with a 5-year survival rate less than $10 \%$. To improve the prognosis of pancreatic cancer, early diagnosis at the resectable stage is essential. Although surgical resection is the only cure for pancreatic cancer, the surgical resection rate is approximately $20 \%-30 \%$, and the early diagnosis of pancreatic cancer still remains a challenge. ${ }^{1}$ Meanwhile, recent improvement of the imaging modalities provides an increasing chance to detect incidental pancreatic lesions ${ }^{2}$ such as pancreatic cysts and pancreatic neuroendocrine tumors (PNETs) and autoimmune pancreatitis. The management of these lesions is completely different

Received: January 8, 2019 Revised: February 12, 2019

Accepted: February 13, 2019

Correspondence: Yousuke Nakai

Department of Gastroenterology, Graduate School of Medicine, The University of Tokyo, 7-3-1 Hongo Bunkyo-ku, Tokyo 113-8655, Japan

Tel: +81-3-3815-5411, Fax: +81-3-5800-9801, E-mail: ynakai-tky@umin.ac.jp ORCID: https://orcid.org/0000-0001-7411-1385

(c) This is an Open Access article distributed under the terms of the Creative Commons Attribution Non-Commercial License (http://creativecommons.org/ licenses/by-nc/3.0) which permits unrestricted non-commercial use, distribution, and reproduction in any medium, provided the original work is properly cited. from that of pancreatic cancer, and it is well known that preoperative misdiagnoses of pancreatic lesions are not uncommon. ${ }^{3}$ Thus, the accurate diagnosis of pancreatic lesions is essential for appropriate treatment selection.

Since its introduction into clinical practice as a new imaging modality, endoscopic ultrasound (EUS) has been utilized in the management of pancreatic neoplasms. ${ }^{4}$ The role of EUS in the management of pancreatic neoplasms includes the imaging diagnosis (identification, characterization, and staging), tissue acquisition, ablation, and drainage for obstructive jaundice or rarely pancreatic duct obstruction. EUS was first utilized in clinical practice because of its high sensitivity for the detection of pancreatic lesions, but soon after its clinical application, EUS-guided fine needle aspiration (EUS-FNA) was reported in 1992 and now plays an important role in the pathological diagnosis of pancreatic lesions. ${ }^{5}$ Meanwhile, in addition to the basic B-mode imaging, several advanced imaging techniques are now available such as the tissue harmonic imaging, ${ }^{6,7}$ contrast-enhanced harmonic imaging, ${ }^{8,9}$ and elastography. ${ }^{10}$ More recently, various EUS-guided interventions are now applicable to pancreatic diseases, celiac plexus neurolysis (CPN), ${ }^{11}$ tumor ablation, ${ }^{12,13}$ and biliary drainage for obstructive jaundice..$^{14,15}$ 
In this review, the current status and the future application of EUS in the management of pancreatic neoplasms will be discussed.

\section{IDENTIFICATION OF PANCREATIC LESIONS}

EUS was first introduced as an imaging modality, and its usefulness over the other imaging modalities is now established. The advantage of EUS lies in its high resolution and its good access to the entire pancreas. When compared to transabdominal ultrasound (AUS), EUS can provide images with high resolution by using high-frequency ultrasound due to its proximity to the pancreas. Furthermore, while the imaging of the head and tail of the pancreas is often hindered by the intestinal gas on AUS, EUS allows the imaging from head to tail end of the pancreas by scanning through the gastric and duodenal wall. Multidetector computed tomography (MD$\mathrm{CT}$ ) is now the standard imaging for pancreatic neoplasms; the usefulness of EUS in comparison with CT is also reported.

In a recent review by Kitano et al., ${ }^{16}$ when AUS and EUS are compared in terms of the detection of pancreatic lesions, the sensitivities of EUS and AUS were $94 \%$ vs. $67 \%$, respectively. The sensitivities of EUS and CT were $98 \%$ and $74 \%$, respectively (Table 1). Furthermore, the advantage of EUS is more prominent in small lesions. In a recent systematic review ${ }^{17}$ of patients with a suspected pancreatic lesion detected by indeterminate CT scan, EUS detected a pancreatic mass with a mean size of $21 \mathrm{~mm}$ in $70 \%$ of the patients. The sensitivity of EUS diagnosis of malignancy was $85 \%$, but the specificity remained as low as $58 \%$. The value of EUS to detect pancreatic cancer in patients with intraductal papillary mucinous neoplasms (IPMNs) $)^{18,19}$ was also reported. The sensitivities of EUS, AUS, CT, and magnetic resonance imaging (MRI) to detect pancreatic cancer were $100 \%, 39 \%, 56 \%$, and 50\%, respectively, in this setting (Table 1).

In summary, despite the recent advancement of cross-sectional imaging modalities, EUS still plays an important role in the detection of pancreatic lesions, especially those too small to be detected by CT.

\section{CHARACTERIZATION OF PANCREATIC NEOPLASMS}

After the detection of pancreatic neoplasms, characterization is the next step. However, it is sometimes difficult to differentiate the type of pancreatic neoplasms only by the basic B-mode imaging. Typically, pancreatic cancer is an ill-defined, hypoechoic, and heterogeneous lesion, while PNET is a well-defined, round, hypoechoic, and homogeneous lesion. As PNET is a hypervascular tumor, hypervascularity is often seen on Doppler EUS. However, those tumors can often show

Table 1. The Diagnostic Yiled of Endoscopic Ultrasound, Computed Tomography, Ultrasound and Magnetic Resonance Imaging in Various Aspects of Pancreatic Lesions

\begin{tabular}{|c|c|c|c|c|}
\hline & EUS & CT & US & MRI \\
\hline \multicolumn{5}{|l|}{ Identification of pancreatic lesions $^{16}$} \\
\hline Sensitivity & $94 \%$ & $74 \%$ & $67 \%$ & $79 \%$ \\
\hline \multicolumn{5}{|l|}{ Detection of cancer in IPMN ${ }^{18}$} \\
\hline Sensitivity & $100 \%$ & $56 \%$ & $39 \%$ & $50 \%$ \\
\hline \multicolumn{5}{|l|}{ Staging of pancreatic cancer } \\
\hline Vascular invasion $^{31}$ & EUS & $\mathrm{CT}$ & & \\
\hline Sensitivity & $88 \%$ & $58 \%$ & - & - \\
\hline Specificity & $93 \%$ & $95 \%$ & - & - \\
\hline Lymph node involvement ${ }^{31}$ & EUS & $\mathrm{CT}$ & & \\
\hline Sensitivity & $58 \%$ & $24 \%$ & - & - \\
\hline Specificity & $85 \%$ & $88 \%$ & - & - \\
\hline Detection of pancreatic lesions in high risk individuals ${ }^{39}$ & EUS & & MRI & \\
\hline Sensitivity for solid lesions & $100 \%$ & - & 0 & - \\
\hline Sensitivity for cystic lesions (any size) & $38 \%$ & - & $89 \%$ & - \\
\hline Sensitivity for cystic lesions ( $<10 \mathrm{~mm})$ & $44 \%$ & - & $88 \%$ & - \\
\hline
\end{tabular}

Modified from Kitano et al., ${ }^{16}$ Kamata et al., ${ }^{18}$ Nawaz et al., ${ }^{31}$ and Harinck et al. ${ }^{39}$

CT, computed tomography; EUS, endoscopic ultrasound; IPMN, intraductal papillary mucinous neoplasm; MRI, magnetic resonance imaging; US, ultrasound. 
atypical imaging findings, and there are some other rare solid lesions, that is, inflammatory tumors, metastatic tumors, and schwannoma. Tissue harmonic echo imaging is now often utilized in clinical practice and is useful in the evaluation of pancreatic cystic lesions. However, its usefulness over the conventional B-mode imaging is reportedly limited in pancreatic solid lesions. ${ }^{7}$

Recently, two image-enhanced EUS functions are clinically utilized, contrast-enhanced harmonic EUS ${ }^{8,20}$ and EUS elastography, ${ }^{21}$ which are reviewed in the following papers in this issue. In addition to these new EUS imaging modalities, needle-based confocal laser endomicroscopy (nCLE) is also reported as a new diagnostic modality for pancreatic lesions under EUS guidance. The diagnostic criteria for pancreatic solid lesions have not been established as compared with those for pancreatic cystic neoplasms. ${ }^{22-25}$ Promising results of nCLE for pancreatic solid lesions were reported in two pilot studies, ${ }^{26,27}$ and typical findings of pancreatic cancer are irregular dark cell aggregates and irregular vessels with puddles of fluorescein within the tumor. Accuracies of nCLE in detecting malignancy were $85 \%$ and $90.9 \%$ in these two studies, respectively. However, a subsequent validation study failed to demonstrate interobserver agreement in nCLE findings. ${ }^{28}$ Thus, the role of nCLE should be further evaluated in the future studies.

Despite the advancement of the abovementioned new EUS-guided imaging, tissue diagnosis is often necessary for the appropriate treatment selection of pancreatic lesions. EUS-guided tissue acquisition, first reported in $1992,{ }^{5}$ provides a sensitivity of $86.8 \%$ and a specificity of $95.8 \%$ in the diagnosis of pancreatic solid lesions. ${ }^{29}$ There is a risk of tumor seeding with EUS-guided tissue acquisition, but the imaging diagnosis alone can misdiagnose pancreatic lesions even in a high-volume center. ${ }^{3}$ In a large-scale study, preoperative EUS-guided tissue acquisition has been increasingly utilized but did not adversely affect survival. ${ }^{30}$ The application of EUS was, in fact, associated with better survival because of the early detection and improved stage-appropriate management. The role of preoperative EUS-FNA is still under debate, but EUS-FNA should be offered when there is doubt in the imaging diagnosis to avoid unnecessary surgical burden to patients with benign lesions such as those in autoimmune pancreatitis. For patients receiving neoadjuvant therapy for pancreatic cancer, which is increasingly administered even in resectable pancreatic cancer, preoperative tissue diagnosis should be established prior to administering neoadjuvant therapy.

\section{STAGING OF PANCREATIC CANCER}

The accurate staging is important for the appropriate treatment selection in patients with pancreatic cancer, which would lead to better survival. For accurate staging in pancreatic cancer, the evaluation of vascular invasion, lymph nodes (LNs), and distant metastasis is necessary (Table 1), and MD$\mathrm{CT}$ is the current standard imaging modality for pancreatic cancer staging whenever possible. Additionally, EUS plays an important role for the staging of pancreatic cancer.

The major vessels to be evaluated in pancreatic cancer are the celiac artery, superior mesenteric artery, and portal vein/ superior mesenteric vein. The presence (or absence) of invasion and its degree determine the resectability of pancreatic cancer; in borderline resectable cases due to vascular invasion, neoadjuvant therapy provides better survival than upfront surgery. In terms of vascular invasion, sensitivity and specificity of EUS are $86 \%$ and $93 \%$, while those of CT are $58 \%$ and $95 \%$, respectively. ${ }^{31}$

The evaluation of LN metastasis on EUS is also reported. ${ }^{4}$ The typical findings of malignant LN on EUS are round, hypoechoic, and size greater than $10 \mathrm{~mm}$. In a systematic review, ${ }^{31}$ the sensitivity and specificity of EUS are $58 \%$ and $85 \%$, respectively, as compared with those of $24 \%$ and $88 \%$ on CT. Although EUS is superior to CT, the sensitivity is still low, and EUS-guided tissue acquisition should be performed if the results change the treatment selection. ${ }^{32}$

Other than LN metastasis, on EUS, the diagnosis of distant metastasis is mainly limited to the left lobe (and a part of the right lobe) of the liver and ascites. EUS diagnosis of small hepatic lesions or a small amount of ascites is useful in conjunction with EUS-guided tissue acquisition. ${ }^{33,34}$ However, EUS cannot detect lesions located far from the upper gastrointestinal lumen such as those in the lung, and CT and EUS are considered as complimentary to each other.

Recently, neoadjuvant treatment is increasingly administered both in resectable and borderline resectable pancreatic cancers. ${ }^{35,36}$ Accurate staging is more important at the time of evaluation after neoadjuvant treatment in this era of neoadjuvant treatment. Investigators have attempted to evaluate tumor response after neoadjuvant treatment using $\mathrm{CT}$, positron emission tomography, and tumor markers, but there has been no established assessment method for the evaluation after neoadjuvant treatment for pancreatic cancer. The role of EUS in this neoadjuvant setting is not fully elucidated and should be further evaluated in the future.

\section{THE ROLE OF EUS IN HIGH-RISK INDIVIDUALS FOR PANCREATIC CANCER}

Early diagnosis of pancreatic cancer is still difficult because 
the surveillance program has not been established yet. Despite its poor prognosis, screening in the general population for pancreatic cancer is not recommended because of its relatively low prevalence. However, screening programs for high-risk individuals ${ }^{37}$ have been increasingly reported. High-risk individuals include familial pancreatic cancer kindred and those with gene mutations such as BRCA2 and PRSS1 mutations. Pancreatic cystic neoplasms are also known as a risk factor for pancreatic cancer ${ }^{38}$ and often subjected to surveillance for pancreatic cancer. Both EUS and MRI had a role in the screening program for high-risk individuals (Table 1). ${ }^{39}$ While MRI can detect small pancreatic cysts, EUS is sensitive for the detection of small solid lesions. In patients with side branch IPMN, EUS reportedly demonstrates high sensitivity for the detection of pancreatic cancer as described above. ${ }^{18,19}$

Therefore, though there are no standard methods for the surveillance program for pancreatic cancer, both EUS and MRI should be incorporated to detect resectable pancreatic cancer and to improve the prognosis of pancreatic cancer among high-risk individuals. ${ }^{40}$

\section{EUS-GUIDED ABLATION}

There are several EUS-guided ablation procedures, for example, CPN, radiofrequency ablation (RFA), and photodynamic therapy (PDT).

$\mathrm{CPN}$ was the first ablation technique under EUS guidance. $\mathrm{CPN}$ has been performed under fluoroscopic or CT guidance, but EUS-guided CPN, first reported in $1996,{ }^{41}$ provides more accurate ablation with safety. Real-time EUS imaging with Doppler function allows better targeting of celiac plexus while avoiding vascular injury. EUS-CPN is shown to provide more persistent pain relief than CT-guided CPN in a randomized controlled trial (RCT). Further improvement in EUS imaging allows the visualization of ganglion, ${ }^{42}$ which can be a target to neurolysis. In a Japanese RCT, ${ }^{43}$ EUS-guided celiac ganglia neurolysis (EUS-CGN) showed better positive response than EUS-CPN ( $73.5 \%$ vs. $45.5 \%, p=0.026)$ with similar adverse events. However, more recently, another RCT ${ }^{44}$ demonstrated no benefits in pain control by EUS-CGN. The study results also suggested that overall survival was shorter in those who received EUS-CGN. Therefore, the role of EUS-CPN/CGN should be further evaluated in the future, especially in the oncological perspective.

There are some techniques of EUS-guided tumor ablation. EUS-guided ethanol ablation was first reported in a patient with hepatic lesions. ${ }^{45}$ EUS-guided ethanol ablation has been reported in patients with PNET, especially those with symptomatic insulinomas who are unfit for surgical resection. ${ }^{46}$
Despite the promising clinical responses, the risk of pancreatitis exists, and the establishment of treatment protocol (the type and amount of injections, the number of session) should be further performed. Use of EUS-guided RFA ${ }^{47,48}$ is increasingly reported for pancreatic neoplasms. Feasibility and safety of RFA are reported for both PNETs and pancreatic cancer. Additionally, a recent advancement of a second-generation photosensitizer led to an increasing interest to PDT in tumors other than cholangiocarcinoma. The feasibility of EUS-PDT was recently reported for solid tumors including pancreatic neoplasms. ${ }^{49}$ Other roles of EUS in pancreatic neoplasms such as brachytherapy and fiducial marker placement are also reported. ${ }^{50}$ EUS-guided injection of antitumor agents, in which various antitumor agents are directly injected into the tumor under EUS guidance, has been investigated since the first report of EUS-guided fine needle injection (EUS-FNI) by Chang et al. in 2000. ${ }^{51}$ Despite promising preliminary results, no RCTs have proved its superiority over the conventional treatment. ${ }^{52}$ However, the concept of direct injection of antitumor agent is attractive, especially for pancreatic cancer, which is often refractory to the conventional chemotherapy, and the new agents for EUS-FNI are still under investigation. ${ }^{53}$ It needs further evaluation to conclude that EUS-FNI is to be a treatment option for pancreatic cancer, either alone or in combination with conventional systemic chemotherapy.

\section{EUS-GUIDED BILIARY DRAINAGE}

Pancreatic cancer often presents with obstructive jaundice, especially those in the head of the pancreas. Transpapillary biliary drainage by endoscopic retrograde cholangiopancreatography (ERCP) is the standard of care for biliary drainage in obstructive jaundice for pancreatic cancer. However, there are some technical and anatomical disadvantages in transpapillary biliary drainage. In addition to failed biliary cannulation, duodenal obstruction in combination with malignant biliary obstruction can be encountered in $20 \%-30 \%{ }^{54}$ EUS-guided biliary drainage (EUS-BD) was first reported as an alternative to percutaneous transhepatic biliary drainage after failed $\mathrm{ERCP}^{55}$ and is now increasingly utilized in the management of pancreatobiliary diseases..$^{56}$ In a systematic review, EUS-BD has similar technical success (odds ratio, 1.78; $p=0.25$ ), better clinical success (odds ratio, $0.45 ; p=0.02$ ), and less adverse events (odds ratio, $0.23 ; p<0.0001$ ) as compared with percutaneous transhepatic biliary drainage. ${ }^{57}$

While biliary cannulation during ERCP can be technically difficult to perform even for experts, biliary access to a dilated bile duct during EUS-BD is often technically easy in cases with obstructive jaundice. There have been some promising 
reports of EUS-BD as a primary biliary drainage method with high technical and clinical success rates and acceptable adverse event rates. ${ }^{58,59}$ In 2018, results of three RCTs ${ }^{60-62}$ of ERCP vs. EUS-BD for malignant biliary obstruction were published. In all studies, technical success rates of EUS-BD were higher than $90 \%$, which were noninferior to those of transpapillary biliary drainage. Furthermore, the rates of adverse events and re-intervention were significantly lower in EUS-BD in one study, ${ }^{61}$ suggesting that EUS-BD can potentially be considered a primary biliary drainage technique for malignant biliary obstruction. These results, however, should be interpreted with caution. First of all, all the studies were conducted in expert centers. EUS-BD is still in the developing phase, and dedicated devices are still lacking. It is well known that a learning curve does exist, and serious adverse events may occur in EUS-BD. ${ }^{63}$ Secondly, the sample size of these three RCTs ${ }^{60-62}$ is too small to confirm the role of EUS-BD as a primary biliary drainage. Finally, in recent years, neoadjuvant treatment has been performed in most of the resectable and borderline resectable pancreatic cancers, and it is still unclear whether EUS-BD can adversely affect clinical outcomes, either surgically or oncologically. Therefore, more studies are warranted, and the standardization of the EUS-BD procedure and devices is also necessary.

\section{CONCLUSIONS}

In summary, EUS plays an important role in the management of pancreatic neoplasms from diagnosis to treatment. While the diagnostic role of EUS is established in processes such as detection, staging, and tissue acquisition, only feasibility and safety have been reported for therapeutic EUS for pancreatic neoplasms. Given the promising results of preliminary studies, the role of therapeutic EUS, which can potentially change clinical practice in the management of pancreatic neoplasms, should be further evaluated.

\section{Conflicts of Interest}

The authors have no financial conflicts of interest.

\section{REFERENCES}

1. Kamisawa T, Wood LD, Itoi T, Takaori K. Pancreatic cancer. Lancet 2016;388:73-85.

2. Mizuno S, Isayama H, Nakai Y, et al. Prevalence of pancreatic cystic lesions is associated with diabetes mellitus and obesity: an analysis of 5296 individuals who underwent a preventive medical examination. Pancreas 2017;46:801-805.

3. Birnbaum DJ, Gaujoux S, Berbis J, et al. Surgery for pancreatic neoplasms: how accurate are our surgical indications? Surgery 2017;162:112-
119.

4. Palazzo L, Roseau G, Gayet B, et al. Endoscopic ultrasonography in the diagnosis and staging of pancreatic adenocarcinoma. Results of a prospective study with comparison to ultrasonography and CT scan. Endoscopy 1993;25:143-150.

5. Vilmann P, Jacobsen GK, Henriksen FW, Hancke S. Endoscopic ultrasonography with guided fine needle aspiration biopsy in pancreatic disease. Gastrointest Endosc 1992;38:172-173.

6. Katanuma A, Isayama H, Bapaye A. Endoscopic ultrasonography using new functions for pancreatobiliary diseases: current status and future perspectives. Dig Endosc 2015;27 Suppl 1:47-54.

7. Matsumoto K, Katanuma A, Maguchi H, et al. Performance of novel tissue harmonic echo imaging using endoscopic ultrasound for pancreatic diseases. Endosc Int Open 2016;4:E42-E50.

8. Kitano M, Kamata K, Imai H, et al. Contrast-enhanced harmonic endoscopic ultrasonography for pancreatobiliary diseases. Dig Endosc 2015;27 Suppl 1:60-67.

9. Kamata K, Takenaka M, Kitano M, et al. Contrast-enhanced harmonic endoscopic ultrasonography for differential diagnosis of localized gallbladder lesions. Dig Endosc 2018;30:98-106.

10. Chantarojanasiri T, Kongkam P. Endoscopic ultrasound elastography for solid pancreatic lesions. World J Gastrointest Endosc 2017;9:506-513.

11. Yasuda I, Wang HP. Endoscopic ultrasound-guided celiac plexus block and neurolysis. Dig Endosc 2017;29:455-462.

12. Lakhtakia S. Therapy of pancreatic neuroendocrine tumors: fine needle intervention including ethanol and radiofrequency ablation. Clin Endosc 2017;50:546-551.

13. Lakhtakia S, Seo DW. Endoscopic ultrasonography-guided tumor ablation. Dig Endosc 2017;29:486-494.

14. Paik WH, Park DH. Endoscopic ultrasound-guided biliary access, with focus on technique and practical tips. Clin Endosc 2017;50:104-111.

15. Minaga K, Kitano M. Recent advances in endoscopic ultrasound-guided biliary drainage. Dig Endosc 2018;30:38-47.

16. Kitano M, Yoshida T, Itonaga M, Tamura T, Hatamaru K, Yamashita Y. Impact of endoscopic ultrasonography on diagnosis of pancreatic cancer. J Gastroenterol 2019;54:19-32.

17. Krishna SG, Rao BB, Ugbarugba E, et al. Diagnostic performance of endoscopic ultrasound for detection of pancreatic malignancy following an indeterminate multidetector CT scan: a systemic review and meta-analysis. Surg Endosc 2017;31:4558-4567.

18. Kamata K, Kitano M, Kudo M, et al. Value of EUS in early detection of pancreatic ductal adenocarcinomas in patients with intraductal papillary mucinous neoplasms. Endoscopy 2014;46:22-29.

19. Kamata K, Takenaka M, Minaga K, et al. Value of additional endoscopic ultrasonography for surveillance after surgical removal of intraductal papillary mucinous neoplasms. Dig Endosc 2018;30:659-666.

20. Lee TH. Can contrast-enhanced harmonic endoscopic ultrasonography differentiate malignancy from benign disease? Clin Endosc 2018;51:5-7.

21. Cui XW, Chang JM, Kan QC, Chiorean L, Ignee A, Dietrich CF. Endoscopic ultrasound elastography: current status and future perspectives. World J Gastroenterol 2015;21:13212-13224.

22. Konda VJ, Meining A, Jamil LH, et al. A pilot study of in vivo identification of pancreatic cystic neoplasms with needle-based confocal laser endomicroscopy under endosonographic guidance. Endoscopy 2013;45:1006-1013.

23. Nakai $\mathrm{Y}$, Isayama $\mathrm{H}$, Shinoura $\mathrm{S}$, et al. Confocal laser endomicroscopy in gastrointestinal and pancreatobiliary diseases. Dig Endosc 2014;26 Suppl 1:86-94.

24. Nakai Y, Iwashita T, Park DH, Samarasena JB, Lee JG, Chang KJ. Diagnosis of pancreatic cysts: EUS-guided, through-the-needle confocal laser-induced endomicroscopy and cystoscopy trial: DETECT study. Gastrointest Endosc 2015;81:1204-1214.

25. Napoléon B, Lemaistre AI, Pujol B, et al. A novel approach to the diagnosis of pancreatic serous cystadenoma: needle-based confocal laser endomicroscopy. Endoscopy 2015;47:26-32. 
26. Giovannini M, Caillol F, Monges G, et al. Endoscopic ultrasound-guided needle-based confocal laser endomicroscopy in solid pancreatic masses. Endoscopy 2016;48:892-898.

27. Kongkam P, Pittayanon R, Sampatanukul P, et al. Endoscopic ultrasound-guided needle-based confocal laser endomicroscopy for diagnosis of solid pancreatic lesions (ENES): a pilot study. Endosc Int Open 2016;4:E17-E23.

28. Karstensen JG, Cârţână T, Constantinescu C, et al. Endoscopic ultrasound guided needle-based confocal laser endomicroscopy in solid pancreatic masses - a prospective validation study. Endosc Int Open 2018;6:E78-E85.

29. Puli SR, Bechtold ML, Buxbaum JL, Eloubeidi MA. How good is endoscopic ultrasound-guided fine-needle aspiration in diagnosing the correct etiology for a solid pancreatic mass?: a meta-analysis and systematic review. Pancreas 2013;42:20-26.

30. Ngamruengphong S, Swanson KM, Shah ND, Wallace MB. Preoperative endoscopic ultrasound-guided fine needle aspiration does not impair survival of patients with resected pancreatic cancer. Gut 2015;64:11051110.

31. Nawaz H, Fan CY, Kloke J, et al. Performance characteristics of endoscopic ultrasound in the staging of pancreatic cancer: a meta-analysis. JOP 2013;14:484-497.

32. Kurita A, Kodama Y, Nakamoto Y, et al. Impact of EUS-FNA for preoperative para-aortic lymph node staging in patients with pancreatobiliary cancer. Gastrointest Endosc 2016;84:467-475.e1.

33. Singh P, Mukhopadhyay P, Bhatt B, et al. Endoscopic ultrasound versus CT scan for detection of the metastases to the liver: results of a prospective comparative study. J Clin Gastroenterol 2009;43:367-373.

34. Levy MJ, Abu Dayyeh BK, Fujii LL, et al. Detection of peritoneal carcinomatosis by EUS fine-needle aspiration: impact on staging and resectability (with videos). Gastrointest Endosc 2015;81:1215-1224.

35. Du L, Wang-Gillam A. Trends in neoadjuvant approaches in pancreatic cancer. J Natl Compr Canc Netw 2017;15:1070-1077.

36. Saito K, Isayama H, Sakamoto Y, et al. A phase II trial of gemcitabine, S-1 and LV combination (GSL) neoadjuvant chemotherapy for patients with borderline resectable and locally advanced pancreatic cancer. Med Oncol 2018;35:100.

37. Canto MI, Hruban RH, Fishman EK, et al. Frequent detection of pancreatic lesions in asymptomatic high-risk individuals. Gastroenterology 2012;142:796-804; quiz el4-el5.

38. Tada M, Kawabe T, Arizumi M, et al. Pancreatic cancer in patients with pancreatic cystic lesions: a prospective study in 197 patients. Clin Gastroenterol Hepatol 2006;4:1265-1270.

39. Harinck F, Konings IC, Kluijt I, et al. A multicentre comparative prospective blinded analysis of EUS and MRI for screening of pancreatic cancer in high-risk individuals. Gut 2016;65:1505-1513.

40. Canto MI, Almario JA, Schulick RD, et al. Risk of neoplastic progression in individuals at high risk for pancreatic cancer undergoing long-term surveillance. Gastroenterology 2018;155:740-751.e2.

41. Wiersema MJ, Wiersema LM. Endosonography-guided celiac plexus neurolysis. Gastrointest Endosc 1996;44:656-662.

42. Levy M, Rajan E, Keeney G, Fletcher JG, Topazian M. Neural ganglia visualized by endoscopic ultrasound. Am J Gastroenterol 2006;101:17871791.

43. Doi S, Yasuda I, Kawakami H, et al. Endoscopic ultrasound-guided celiac ganglia neurolysis vs. celiac plexus neurolysis: a randomized multicenter trial. Endoscopy 2013;45:362-369.

44. Levy MJ, Gleeson FC, Topazian MD, et al. Combined celiac ganglia and plexus neurolysis shortens survival, without benefit, vs plexus neurolysis alone. Clin Gastroenterol Hepatol 2019;17:728-738.e9.

45. Barclay RL, Perez-Miranda M, Giovannini M. EUS-guided treatment of a solid hepatic metastasis. Gastrointest Endosc 2002;55:266-270.
46. Park DH, Choi JH, Oh D, et al. Endoscopic ultrasonography-guided ethanol ablation for small pancreatic neuroendocrine tumors: results of a pilot study. Clin Endosc 2015;48:158-164.

47. Song TJ, Seo DW, Lakhtakia S, et al. Initial experience of EUS-guided radiofrequency ablation of unresectable pancreatic cancer. Gastrointest Endosc 2016;83:440-443.

48. Choi JH, Seo DW, Song TJ, et al. Endoscopic ultrasound-guided radiofrequency ablation for management of benign solid pancreatic tumors. Endoscopy 2018;50:1099-1104.

49. Choi JH, Oh D, Lee JH, et al. Initial human experience of endoscopic ultrasound-guided photodynamic therapy with a novel photosensitizer and a flexible laser-light catheter. Endoscopy 2015;47:1035-1038.

50. Han J, Chang KJ. Endoscopic ultrasound-guided direct intervention for solid pancreatic tumors. Clin Endosc 2017;50:126-137.

51. Chang KJ, Nguyen PT, Thompson JA, et al. Phase I clinical trial of allogeneic mixed lymphocyte culture (cytoimplant) delivered by endoscopic ultrasound-guided fine-needle injection in patients with advanced pancreatic carcinoma. Cancer 2000;88:1325-1335.

52. Herman JM, Wild AT, Wang H, et al. Randomized phase III multi-institutional study of TNFerade biologic with fluorouracil and radiotherapy for locally advanced pancreatic cancer: final results. J Clin Oncol 2013;31:886-894.

53. Nishimura M, Matsukawa M, Fujii Y, et al. Effects of EUS-guided intratumoral injection of oligonucleotide STNM01 on tumor growth, histology, and overall survival in patients with unresectable pancreatic cancer. Gastrointest Endosc 2018;87:1126-1131.

54. Nakai Y, Hamada T, Isayama H, Itoi T, Koike K. Endoscopic management of combined malignant biliary and gastric outlet obstruction. Dig Endosc 2017;29:16-25.

55. Giovannini M, Moutardier V, Pesenti C, Bories E, Lelong B, Delpero JR. Endoscopic ultrasound-guided bilioduodenal anastomosis: a new technique for biliary drainage. Endoscopy 2001;33:898-900.

56. Nakai Y, Isayama H, Yamamoto N, et al. Indications for endoscopic ultrasonography (EUS)-guided biliary intervention: does EUS always come after failed endoscopic retrograde cholangiopancreatography? Dig Endosc 2017;29:218-225.

57. Sharaiha RZ, Khan MA, Kamal F, et al. Efficacy and safety of EUS-guided biliary drainage in comparison with percutaneous biliary drainage when ERCP fails: a systematic review and meta-analysis. Gastrointest Endosc 2017;85:904-914.

58. Hara K, Yamao K, Niwa Y, et al. Prospective clinical study of EUS-guided choledochoduodenostomy for malignant lower biliary tract obstruction. Am J Gastroenterol 2011;106:1239-1245.

59. Hara K, Yamao K, Hijioka S, et al. Prospective clinical study of endoscopic ultrasound-guided choledochoduodenostomy with direct metallic stent placement using a forward-viewing echoendoscope. Endoscopy 2013;45:392-396.

60. Bang JY, Navaneethan U, Hasan M, Hawes R, Varadarajulu S. Stent placement by EUS or ERCP for primary biliary decompression in pancreatic cancer: a randomized trial (with videos). Gastrointest Endosc 2018;88:9-17.

61. Paik WH, Lee TH, Park DH, et al. EUS-guided biliary drainage versus ERCP for the primary palliation of malignant biliary obstruction: a multicenter randomized clinical trial. Am J Gastroenterol 2018;113:987997.

62. Park JK, Woo YS, Noh DH, et al. Efficacy of EUS-guided and ERCP-guided biliary drainage for malignant biliary obstruction: prospective randomized controlled study. Gastrointest Endosc 2018;88:277-282.

63. Poincloux L, Rouquette O, Buc E, et al. Endoscopic ultrasound-guided biliary drainage after failed ERCP: cumulative experience of 101 procedures at a single center. Endoscopy 2015;47:794-801. 